This report was prepared as an account of work sponsored by an agency of the United States Government. Neither the United States Government nor any agency thereof, nor any of their employees, makes any warranty, express or implied, or assumes any legal liability or responsibility for the accuracy, comple!eness, or usefulness of any information, apparatus, product, or process disclosed, or represents that its use would not infringe privately owned rights. Reference herein to any specific commercial product, process, or service by trade name, trademark, manufacturer, or otherwise does not necessarily constitute or imply its endorsement, recom. mendation, or favoring by the United States Government or any agency thereof. The views and opinions of authors expressed herein do not necessarily state or reflect those of the United States Government or any agency thereof.

SIMULATION OF TRANSVERSE COMBINING OF SPACE-CHARGE DOMINATED BEAMS*

LBL -21349

DE86 015125

C. M. Celata

\author{
Lawrence Berkeley Laboratory \\ University of California \\ Berkeley, CA 94720
}

July 1986

* This work was supported by the Offi:e of Energy Research, Office of Basic Energy Sciences, U.S. Department of Energy, under Contract No. DE - ACO3-76SF00098. 


\title{
SIMULATION OF TRANSVERSE COMBINING OF SPACE-CHARGE DOMINATED BEAMS*
}

\author{
C. M. Celata \\ Lawrence Berkeley Laboratory \\ University of California, Berkeley, CA 94720
}

\begin{abstract}
Rms emittance growth in the transverse plane due to the transverse combining of four identical elliptical beams of uniform density has been investigated. The emittance growth can be related by conservation of energy to the change in the electrostatic field energy. Its dependence on initial beam positions and radii has been calculated analytically for round beams and by computer simulation for elliptical beams.
\end{abstract}

\section{INTRODUCTION}

Limitations on the charge per unit length which can be focused suggest that the ion beam for heavy ion fusion be transported as separately focused multiple beams at the low energy end of the accelerator. When the energy of the beams is large enough, it may be more cost-effective to combine several of them. In this paper the transverse combining of four such beams is considered, with the goal of selecting initial conditions which will minimize transverse rms emittance growth. Only transverse dynamics are considered -- longitudinal fields are ignored -- and the beam is assumed to be coasting, with no spread in longitudinal energy.

A system for combining beams might consist of a matching section, where the shape of the beams if tailored to minimize emittance growth during the combining process; a bending section, where the beams' centroid trajectories are deflected to decrease the distance between beams; and a merging section, where the bearns "see" each other and form a single beam. In this paper we consider only the merging section. The merging is assumed to occur in a focusing lattice which may be alternating-gradient or constant focusing.

We will measure the growth of the one dimensional rms emittance using the ratio of the final emittance to the total initial phase space area in that dimension, i.e., $c_{f_{x}} /\left(2 c_{j x}\right)$, where $c_{i x}$ and $c_{f x}$ are the initial single beam emittance and the final emittance in the $x$ direction. This quantity can be written as

$$
H_{X} \frac{c_{f X}}{2 c_{1 X}}=\left(\frac{c_{O X}}{2 c_{1 X}}\right)\left(\frac{c_{f X}}{c_{O X}}\right) \text {. }
$$

where $c_{0}$ is the emittance of the composite beam when the initial beams flrst see each other. The first factor in Eq. (1) is due to the fact that empty spaces between the beams flll with particles, adding to the phase

- This work was supported by the Otfice of Energy Research, Office of Basic Energy Sclences, Department of Energy under Contract No. DE-ACQ4-765F00098. 
space occupied by the beam. This "geometric" emittance dilution would be the only increase in emittance for low intensity beams. The second factor is the phase space dilution caused by space charge (conversion of electrostatic field energy to transverse kinetic energy) and, if the rms radii of the beam change, by work done by the focusing fields. Simulation has shown that because space charge couples the transverse dimensions, the final state of the beam tends to have equal emittance in the two planes. This has also been observed experimentally in the SBTE at Lawrence Berkeley Laboratory for a single space-charge-dominated beam. I Then if the rms beam radii are not changed during the merging, conservation of energy gives the result:

$$
c_{f x}=\sqrt{\frac{\left\langle x^{2}\right\rangle c_{o x} / c_{o x}{ }^{2}+\left\langle y^{2}\right\rangle-\left\langle y^{2}\right\rangle \Delta U / T_{o x}}{\left\langle x^{2}\right\rangle+\left\langle y^{2}\right\rangle}}
$$

and similarly for $y$, where $U$ is the total electrostatic field energy per unit length (beam and surrounding vacuum), $\Delta U=U_{f}-U_{i}$, and $T_{o x}$ is the total $x$ kinetic energy per unit length for the four beams. This is a general result for the change in emittance when the spatial configuration of a beam is changed, and reduces to the result of Wangler ${ }^{2}$ for the case of azimuthal symmetry. If the rms beam radii change during the process, the wark done by the focusing fields can be added to Eq. (2). For constant focusing, for instance, with focusing force $F_{x}=-k_{x}, \Delta U$ in Eq. (2) would then become $\Delta U+N k_{x} \Delta a 2 / 2$, where " $a$ " is the beam radius and $N$ is the number of particles per unit length.

This paper will consider the combining of four identical beams which are upright ellipses in configuration space, and have uniform density and gaussian velocity distributions before the merge. The initial rms $x$ and $y$ momenta for each beam are spatially uniform, and the $x$ and $y$ emittances are equal. Initially (when the beams first "see" each other) the beam centroid positions ar $;(x, y)=\left(\delta_{x}, \delta_{y}\right),\left(-\delta_{x}, \delta_{y}\right), \quad\left(\delta_{x},-\delta_{y}\right)$, and $\left(-\delta_{x},-\delta_{y}\right)$, with the origin at the vacuum chamber axis and the quadrupoles on the $x$ and $y$ axes in the case of AG focusing (see Fig. 1). The boundary condition is a perfectly conducting pipe of radius $R$. We consider first the simple case of four round beams of radius "a" with $\delta_{x}=\delta_{y}=\delta / \sqrt{2}$.

\section{COMBINING ROUND BEAMS}

Wangler $^{2}$ has indicated that the equilibrium state for a space-charge-dominated beam is likely to have a uniform density. Anderson ${ }^{3}$ has shown this profile to be a minimum of the electrostatic field energy for a one dimensional (sheet) beam. This neglects the sheath at the beam edge, but the sheath width is of the order of the Debye length, $\lambda_{D}$, and $\lambda_{0} \ll$ a for space-charge-dominated beams. We will assume then that the beam evolves after the merge to a state with uniform density. Further, since the Initial configuration and assumed final beam state both follow the same rms envelope equation, we set the Initial rms radius equal to its matched value, and assume the final beam will then be matched, with the ame rms radius. Use of a partlcle-in-cell simulation code shows both 


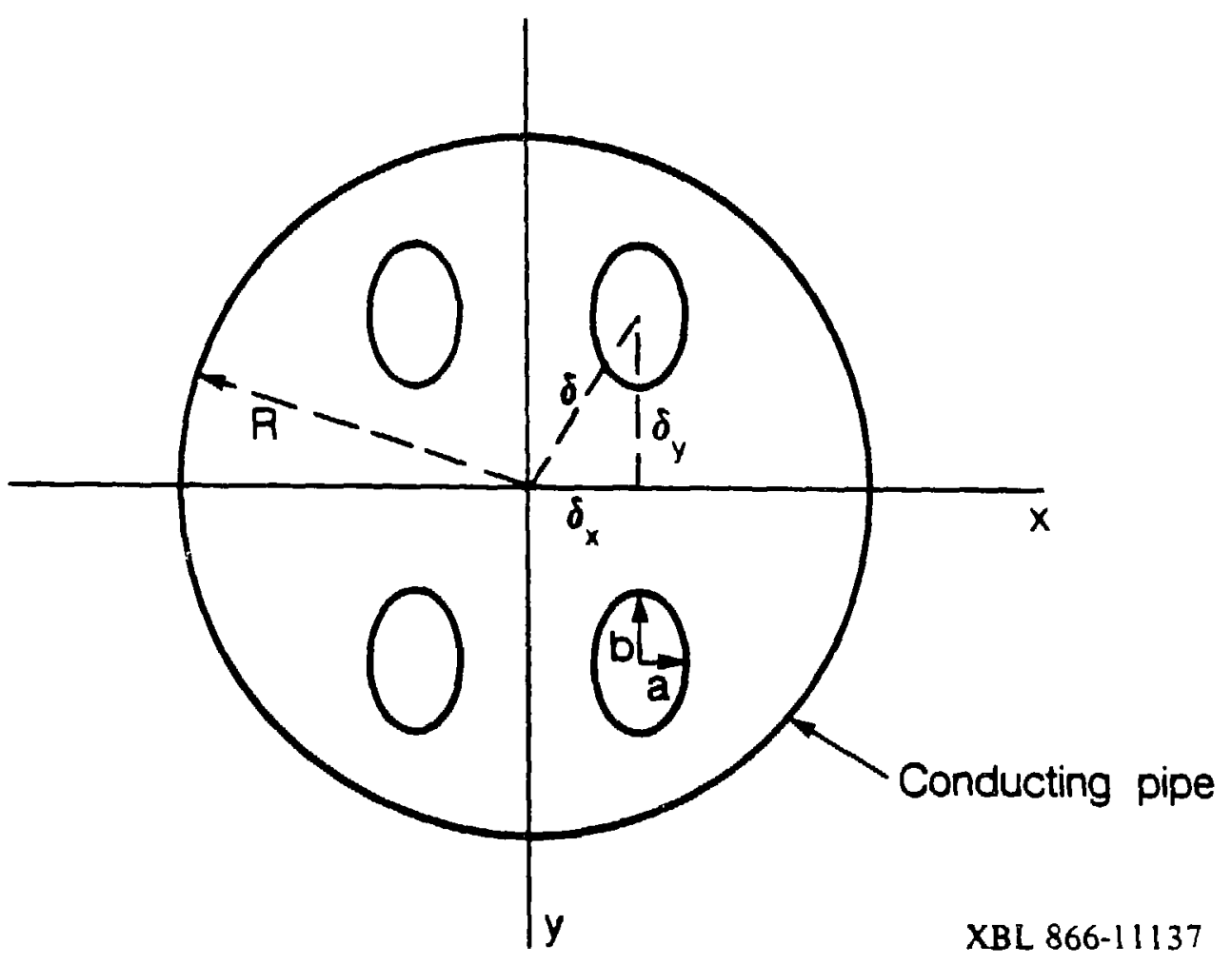

Fig. 1. Transverse plane geometry showing four beams when they first "see" each other.

assumptions to be true only for small $8 / a, \delta / a<2$. For larger $\delta / a$, the ims radius of the beam oscillates and particle loss occurs. With these assumptions,

$$
\Delta U=\frac{\mu^{2} q^{2}}{16}\left[3-4 \ln \frac{1-a^{8}}{4}\left(\frac{a}{\delta}\right)^{3}\left(1+2 \frac{\delta^{2}}{a^{2}}\right)^{2}\right] \text {. }
$$

where $g=\delta / R$. Note that $U_{i}, U_{f}$, and therefore $\Delta U$, will always be proportional to the total charge per unit length squared, regardless of the beam shape, orientation, or density profile. This is the only dependence of $M$ on Nq. Since $g^{8}$ is generally much less than unity, $\Delta U$ essentially depends only on $\delta / a$ and Nq. $M_{x}$ can now be calculated from Eqs. (1), (2), and (3), since $c_{o x} /\left(2 c_{i x}\right)=0.5 \sqrt{1+2 \delta 2 / a Z}$.

We can use these results to determine desirable qualities for beam merging geometries. We will keep constant the emittance of the original beams, so that $T_{o x} \propto a^{-2}$, and also the charge per unit length, in order to study scaling with geometrical factors. We might wish to hold $\delta / a, \delta-a$, or $\delta$ constant for practical experimental reasons. If $\delta / \mathrm{a}$ is kept constant, $M_{x}$ increases monotonically with "a". This reflects the fact 
that $\Delta U$ is unchanging, but the additional transverse kinetic energy it supplies makes a larger relative change in the total when the beam is colder. The results when the clearance between beams is held constant, to allow, for instance, for a septum in the bending section, are shown in Fig. 2

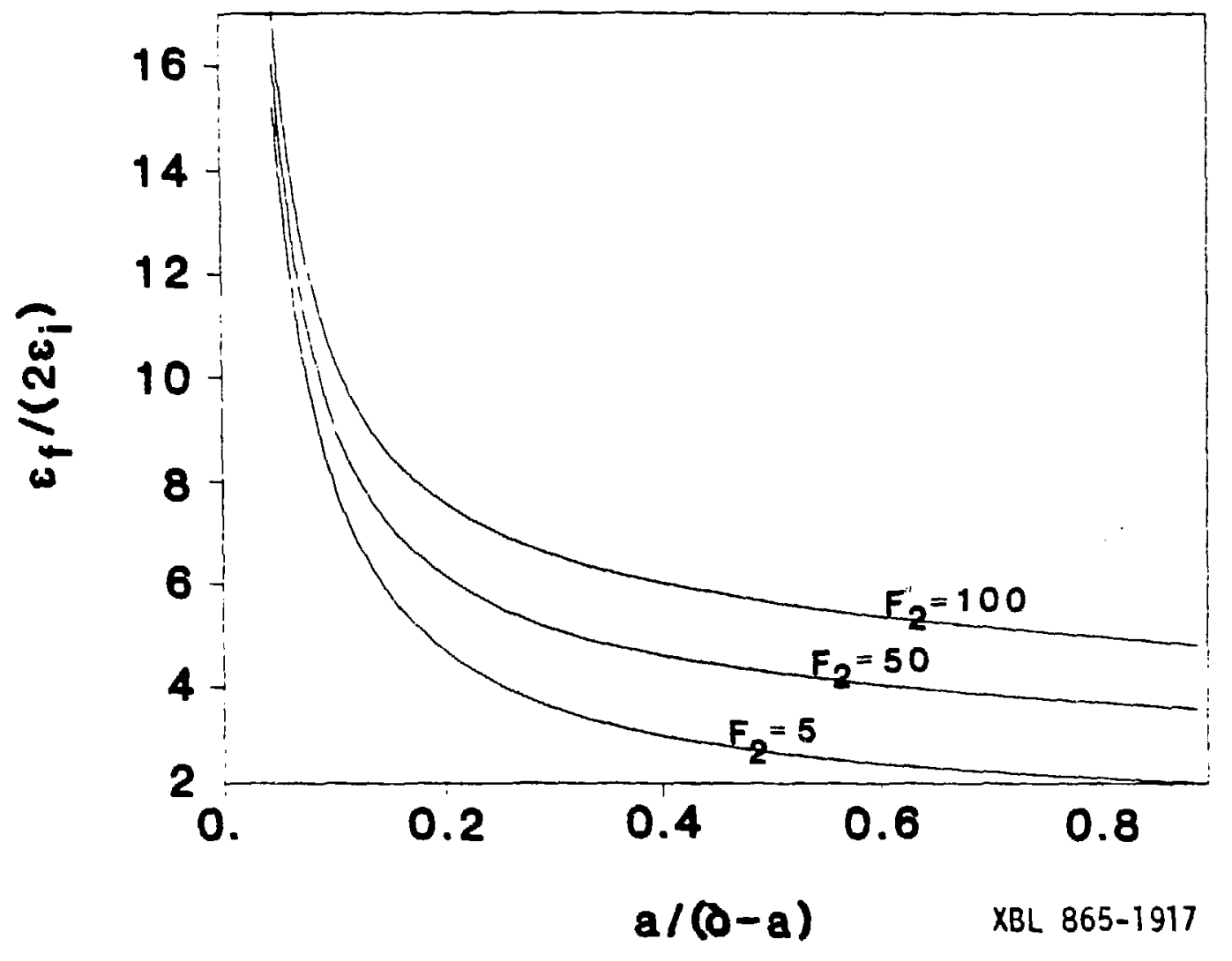

Fig. 2. One dimensional emittance growth for round beams with constant clearance, $\delta-a$, and varying "a". (s-a) $/ R=0.284$. $\quad F_{2}=$ $\left(\left(\sigma^{2}-\sigma^{2}\right) / \sigma^{2}\right)(\delta-a) 2 / a 2$, where $\sigma$ and $\sigma_{0}$ are the betatron phase advance per lattice period with and without space charge for a uniform beam with the same charge per unit length and $x$ and $y$ kinetic energy as the initial conflguration.

for a typical set of parameters. Note that the monotonic decrease of $\boldsymbol{H}_{\mathbf{x}}$ in Fig. 2 is not representative of its behavior at all values of the clearance, as can be seen from Eqs. (1)-(3). $H_{x}$ decreases with increasing "a" if $\delta$ is held constant and "a" is increased (Fig. 3). In both of these cases, as " $a$ " increases, $\Delta U$ and $T_{\text {ox }}$ both decrease. Of course for constant beam radius, $M_{x}$ monotonically Increases with $\delta / a$ and Nq. Finally, we might wish to fix the radius of the final beam. Then if $\delta$ increases, "a " must decrease. In this case $M_{x}$ Increases monotonically with decreasing "a", as the space charge dllution factor decreases, while the area dilution increases. 


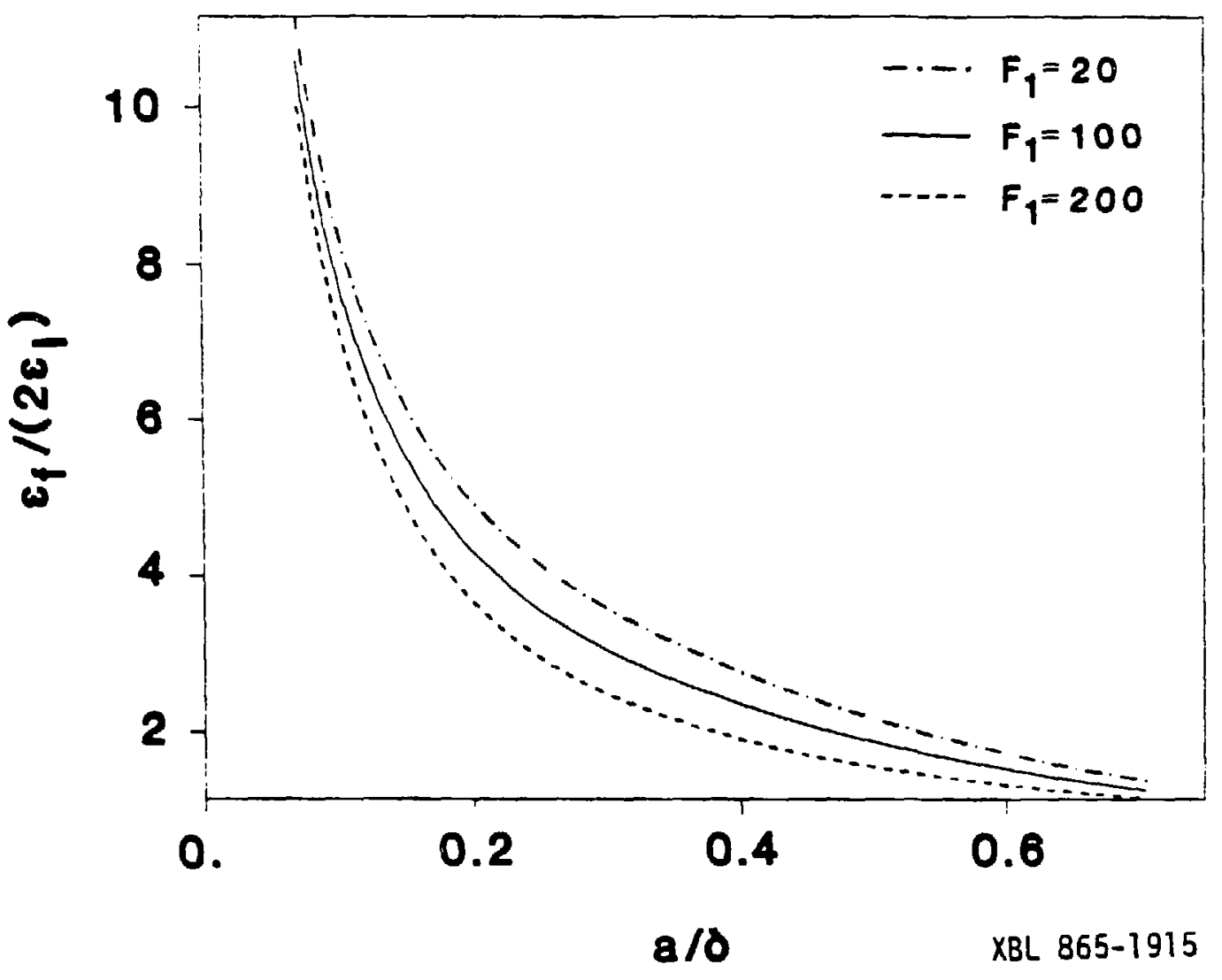

Fig. 3. One dimensional emittance growth for round beams with constant d. $\delta / R=0.506$. $\quad F_{1}=\left(\left(\sigma^{2}-\sigma^{2}\right) / \sigma^{2}\right)\left(\delta^{2} / a^{2}\right)$. See Fig. 1 for definitions of $a$ and $\sigma_{0}$.

\section{COMBINING FOUR ELLIPTICAL BEAMS}

The two dimensional particle-in-cell simulation code SHIFTXY has been used to compute the quantity $c_{f} / c_{0}$ for the case of four elliptical beams described in the Introduction. Alternating gradient focusing was produced using a thin lens approximation. For all runs a focusing system which provided a single particle phase advance of $60^{\circ}$ per lattice period was used. Emittance of the initial single beams was correct to give a space charge depressed phase advance of $20^{\circ}$ per lattice period in a $60^{\circ}$ lattice upstream of the merge. Again the attempt was made to rms-match the beam. The rms radii of the initial configuration were set equal to those of a matched uniform beam with the same charge per unit length, since this was the assumed final state. Therefore the initial $\left\langle x^{2}\right\rangle /\left\langle y^{2}\right\rangle$ was 2.79 for all runs. The initial value of $x_{r m s} / R$ was 0.35 .

For $\delta_{x} / a=\delta_{y} / b$, where $a$ and $b$ are the initial beams' major and minor radil, simulation results for $M_{x}$ and $M_{y}$ had the values which would be calculated using the value of $\Delta U$ given in Eq. (3) for round beams, with $\delta=\sqrt{2} \delta_{x}$. (Runs were done for $1 \leq \delta_{x} / a \leq 2.5$.) Therefore the quantity $\zeta=\left(\delta_{x} / a\right) /\left(\delta_{y} / b\right)$ would seem to be of interest, as a "shape 
factor" characterizing the departure of the behavior of the system from that of round bearns $(\zeta=1)$. The geometry of the initial configuration is then completely specified by $\zeta, \delta_{x} / a$, and $\left(x_{r m s} / y_{r m s}\right)$. A brief investigation of the dependence of $M_{x}$ and $M_{y}$ on the first two of these parameters was done, but much remains to be studied. The results are shown in Figs. 4-6. The iritial rms radii of the configuration, single beam emittances, and focusing system were held constant for all runs. Therefore as $\delta_{x} / a$ increased, " $a$ " decreased, and $T_{o x}$ increased.

As shown in Fig. 4, matching was again a problem for configurations where the beams were not almost touching. For these cases the final beam was also not uniform after - 100 lattice periods, even though the emittance growth seemed to have ceased. $\left\langle x^{4}\right\rangle \mid\left\langle x^{2}\right\rangle 2$ was larger than its value for a uniform beam, indicating probably some hollowing of the beam. Beam loss also occurred for $\delta_{x} / a \geqslant 2$, and increased with $\delta_{x} / a$. For the case of Figs. 4 and 5, 5\% of the beam was lost in 150 lattice periods. For $\delta_{x} / a=2.5, \zeta=1,13 \%$ was lost in 100 periods. Figure 5 demonstrates that, as shown computationally by Wangler ${ }^{2}$ and analytically by Anderson, 3 most of the emittance growth due to the change in the electrostatic field energy occurs within a fraction of a plasma period, though subsequently the $x$ and $y$ emittances equilibrate.

Though the range of parameters spanned was limited by computer time constraints (the small hot beams for large $\delta_{x} / a$ require extremely good spatial and temporal resolution) the results given in Fig. 6 indicate that, as expected, emittance growth increases with $\delta_{x} / a \ldots$ the initial

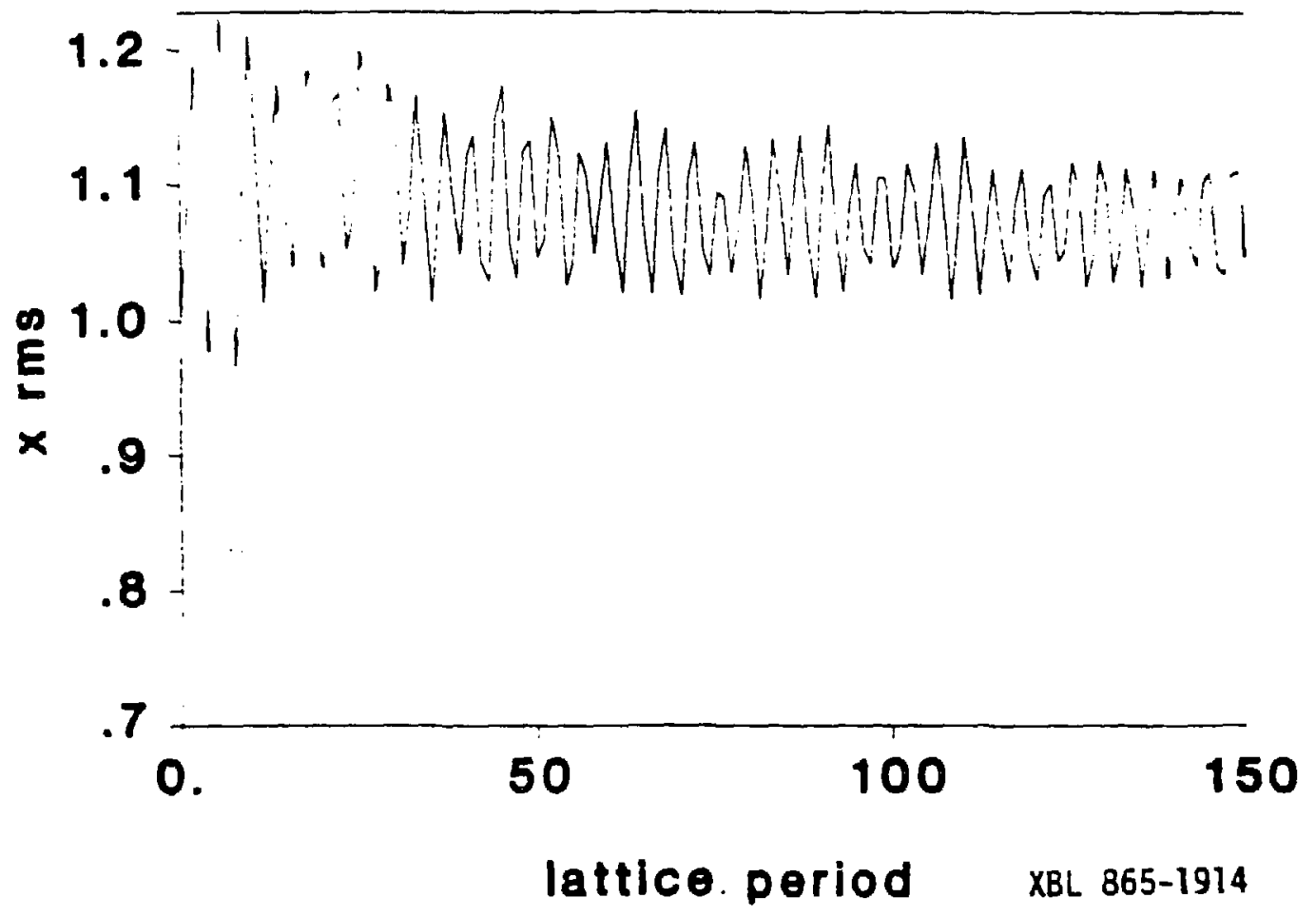

Fig. 4. $x_{\text {sms }}$ vs. $z$ for $\zeta=1, \delta_{x} / a=2$. Other parameters are given in the text. 


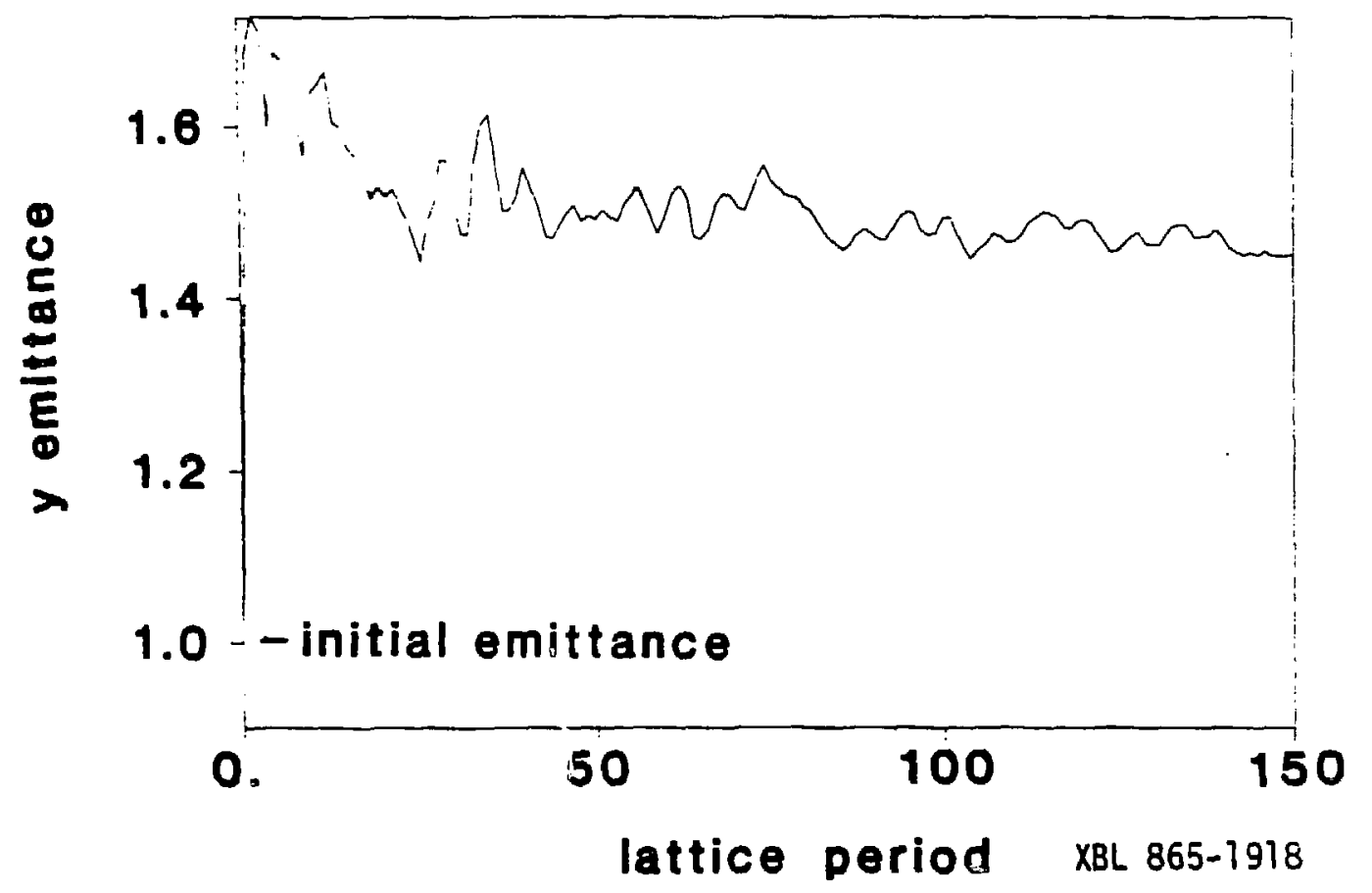

Fig. 5. $c_{f} / c_{\text {oy }}$ vs. $z$ for $\zeta=1, \delta_{x} / a=2$. Other parameters are given in the text.

configuration should be as close as possible to a uniform beam to minimize emittance growth. $\zeta=2$ gives lower emittance growth than $\zeta=1$ for the same $\delta_{x} / a$. As seen from a comparison of the dashed and solid curves in Fig. 6, for the $x$ dimension this is because the space charge dilution factor is lower, while for $y$ the area dilution factor is lower than for $\zeta=1$. Note that $M_{x}=M_{y}$ because $c_{f x}=c_{f y}$.

\section{MATCHING}

As described above, an attempt was made to rms-match the merging beams using the rms envelope equation:

$$
\frac{d^{2} x r m s}{d z^{2}}+k_{x} x_{r m s}-\frac{c_{x}^{2}}{x_{r m s}^{2}}-\frac{q}{m r^{3} v_{z}^{2}} \frac{\left\langle x E_{x}\right\rangle}{x_{r m s}}=0 .
$$

For the case of four round beams with $\delta_{x}=\delta_{y}$, the last term in Eq. (4) has the same value as for a uniform round beam, since in both cases $\left\langle x E_{x}\right\rangle=\mathrm{Mq} / 2$. Therefore, if the initial configuration were rms-matched, and the final beam were uniform, one might hope that the beam would stay matched throughout the merging process However, for $\delta / a \geqslant 2$, asclllations of the rms radli were seen at all 2 , with an amplitude which decreased with increasing $z$. This demonstrates a limitation on the use of the rms envelope equation for matching during time develogment of the 


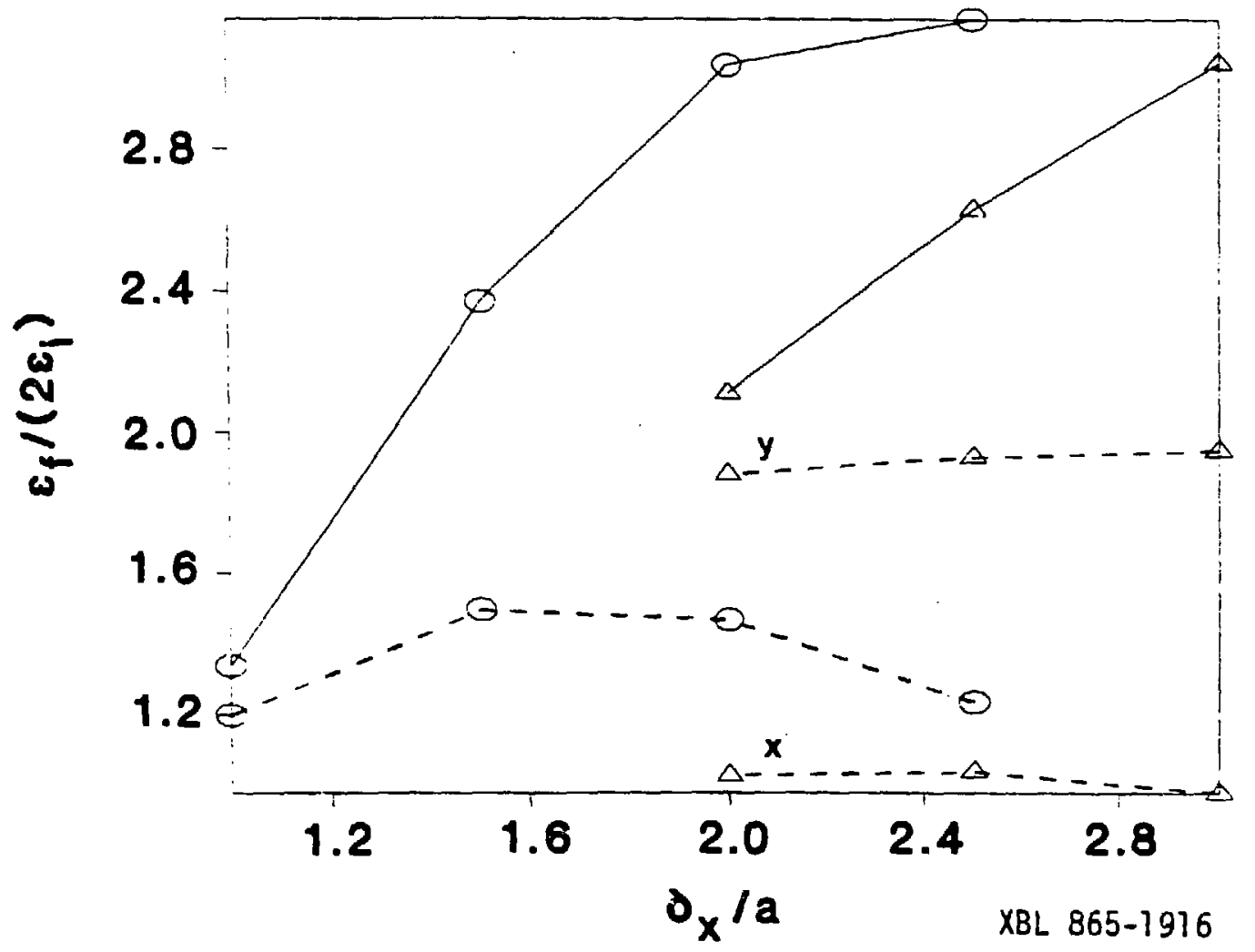

Fig. 6. One dimensional rms emittance growth vs. $\delta_{x} / a$ for $\zeta=1$ and $\zeta=2$, computed by S:-IIF TXY. Circles and triangles are simulation results for $\zeta=1$ and $\zeta=2$ respectively. Solid lines are total emittance growth, $c_{f x} /\left(2 c_{i x}\right)$, while dashed lines are the space charge dilution factor only.

distribution function. Note that as the merging progressed, the emittance term in the envelope equation remained negligible compared to the space charge term, so that the changing emittance was not important in matching the beam.

For elliptical beams in an AG focusing system a similar result was found. The matched rms radii appeared to be a function of $z$, changing as the dlstribution function evolved.

\section{CONCLUSIONS}

Transverse rms emittance growth due to the transverse combining of Identical uniform elliptical beams with gaussian velocity distribution and uniform $x$ and $y$ rms momenta has been investigated. For round beams the "space charge dilution factor" depends on $\delta / a$ and the Initial beam kinetic energy, with negligible dependence on the vacuum chamber radius. For elliptical beams, simulations show that round beam results can be used to calculate emittance growth for $\delta_{x} / a=b_{y} / b, 1 . e ., \zeta=1$. Some results for other cases $(\zeta=2)$ have been given. The one dimensional relative emitiance growth, $c_{f x} /\left(2 c_{i x}\right)$, for experimentally reasonable 
conditions can be made $\approx 2$. For cases where the beams were not almost touching each other, matching using the rms envelope equation was shown to present a problem, since $\left\langle x E_{x}\right\rangle$ and $\left\langle y E_{y}\right\rangle$ vary with time. In such situations beam loss occurred, and the beam did not settle down to a uniform final density profile within the 100-150 lattice periods for which the simulation code was run.

\section{ACKNOWLEDGEMENTS}

The author would like to acknowledge very helpful discussions with $D$. Judd, L.J. Laslett, L. Smith, and especially A. Faltens.

\section{REFERENCES}

1. Michael G. Tiefenback, "Space-charge Limits on the Transport of Ion Beams in a Long Alternating Gradient System", Lawrence Berkeley Laboratory report LBL-21611, 1986.

2. T.P. Wangler, K.R. Crandall, R.S. Mills, and M. Reiser, IEEE Trans. Nucl. Sci., 32, 2196 (1985).

3. D.A. Anderson, "Internal Dynamics and Emittance Growth in Non-Uniform Beams", accepted for publication in Particle Accelerators.

4. F.J. Sacherer, IEEE Trans. Nucl. Sci. 18, 1105 (1971). 
This report was done with support from the Department of Energy. Any conclusions or opinions expressed in this report represent solely those of the author(s) and not necessarily those of The Regents of the University of California, the Lawrence Berkeley Laboratory or the Department of Energy.

Reference to a company or product name does not imply approval or recommendation of the product by the University of California or the U.S. Department of Energy to the exclusion of others that may be suitable. 